\title{
The Proposal of Metrics and Evaluation Methods to Explicit Knowledge of the Absorptive Capacity
}

\author{
Ken Kaminishi \\ Graduate School of Innovation and Technology Management, \\ Yamaguchi University \\ Address: 1677-1 Yoshida, Yamaguchi, 753-8511 \\ E-mail: kaminisi@yamaguchi-u.ac.jp \\ Yoshiyuki Matsuura \\ Graduate School of Innovation and Technology Management, \\ Yamaguchi University \\ E-mail: matu@yamaguchi-u.ac.jp \\ Kenji Miyake \\ Graduate School of Science and Engineering, \\ Yamaguchi University \\ E-mail: s011wc@yamaguchi-u.ac.jp
}

\begin{abstract}
The importance of Absorptive Capacity has been stated in many previous researches. When small and medium-sized enterprises with poor management resources perform product development with an outside-in open innovation approach, one of the important factors determining the success or failure is Absorptive Capacity of the licensing-in company that form a foundation of technology learning. In this paper, we propose a method for evaluating the Absorptive Capacity of the company, and it is intended to propose a method for utilizing Absorptive Capacity as a measurement of "readiness" to external knowledge.
\end{abstract}

Keywords: Absorptive Capacity, External knowledge, Open Innovation

Paper received: 07/12/2013

Paper accepted for Publication: 31/01/2014 


\section{INTRODUCTION}

Since the open innovation is advocated by Chesbrough (2003), the promotion of innovation by utilizing external knowledge, including industry-university cooperation is in the spotlight more and more in Japan. Outside-in open innovation, that is to say licensing of technology itself is widely being used among large corporations in Japan, for the Japanese company where it is supposed that it has an independent management tendency about technical development (Not invented here; NIH syndrome), an accepted technology has a high possibility that only a restrictive and complementary role will be given. Therefore, the way of organization appropriate in open innovation era is explored. (Motohashi et al., 2012).

One of the major important factors that determine the success or failure of the outsidein open innovation is Absorptive Capacity $(A C)$ of the licensee company that form a foundation of technology learning (Veugelors and Cassiman, 1999). Since AC was applied to microanalysis by Cohen and Levinthal(1989,1990), although many researchers have studied AC theoretically and empirically, it is claimed that the original meaning of $A C$ is becoming ambiguous through these studies (Lane et al., 2006; Volberda et al., 2010) and a redefinition is tried (Zahra and George, 2002; Todorova and Dursin, 2007). However, an investment for activities of all studies that focus on $\mathrm{AC}$ is common in that the value to the organization of external knowledge is influenced (Fabrizio, 2009), and as the definition of Cohen and Levinthal (1990) has been widely accepted most (Murovec and Prodan, 2009), in this paper, we define AC as "the ability of an organization to recognize the value of new, external information, assimilate it, and apply it to commercial ends".

Many previous studies have been to clarify the factors that improve AC of an organization. As a typical factor, they can cite in-house R\&D linvestment (Cohen and Levinthal, 1989), skills that employees possess (Vining, 2006), past joint R\&D experiences (Becker and Diaz, 2004), routine of the organization (Zahra and George, 2002) and organizational culture (Van Den Borch et al., 1999) etc. .

As given condition which is with an effect of $A C$ to give innovation and a factor to influence AC accumulation, to apply to decision making to introduce a concept of AC into actual external knowledge, it is necessary to be operationalized to derive an answer to questions such as the following: "Given the current status of our own company, what is the activity to contribute to improvement of AC most?" or "As for the premise of our current AC, is it possible to implement innovation that utilize an external knowledge?". We thus believe a framework that provides guidance to those questions would increase the possibility to exploit the concept of $A C$ as management techniques. However, $A C$ is essentially qualitative and so far as we know, it cannot be said that the trial of quantification is performed enough until now. In this paper, we have devised a framework for assessing this question, and it is intended to propose a method for utilizing AC as a measurement of "readiness" of its company to external knowledge.

We think that AC quantification is especially suggestive when SMEs with poor management resources aim at innovation which utilized external knowledge. AC is cumulative, and it is said that it is also important to have the broadness of knowledge (Cohen and Levinthal, 1990). As for this, from a viewpoint of accumulation of AC, it is thought that the possibility that SMEs have advantages for large enterprises with use of external knowledge is extremely low, if other conditions are set the same. In other words, SMEs which are implicitly inferior to AC are considered to mean that working as a creator is effective as an accepting person of knowledge. 
The uneven distribution of $A C$ depending on the corporate scale can support progress of the inside-out open innovation by SMEs theoretically, but the outside-in opening innovation by SMEs does not suggest the situation becoming effective in it alone.

In order for SMEs which face a resource constraint to compete for big-firms, a network with outside plays a decisive role (Dodgson and Rothwell, 1994), in addition, it is apparent that the level of AC is an element which is more important to innovation by SMEs so that it is insisted when an actor connecting the company and the outside environment is assets which are important to SMEs (Noteboom, 1998). Furthermore, as represented in the cluster policy, it is strongly desirable from the point where the outside-in innovation by SMEs, the growth of the company by it will lead to the development of local economy (Maskell and Malmberg, 1999). In Japan, in spite of many political activities, the penetration into SMEs of industry-university collaboration, which is a representational approach of the outside-in innovation, does not reach sufficiently level.

Madrid-Guijarro et al. (2009) states that the human and financial barriers have a negative correlation with innovation in SMEs, additionally and they tend to hesitate to funding from outside due to the high risk of innovation. Innovations that utilizes external knowledge, and if the risk is higher (the cognitive theory at least) than when based on the knowledge that was created internally, it is expected that SMEs become more cautious in practical use of external knowledge. If we assume this, in order to promote efforts of outside-in open innovation by SMEs, we consider that it becomes important to show objectively whether innovation that utilized external knowledge accompanies allowable risk assuming the company's AC.

In this paper, we describe the evaluation indices and methods for AC which is basically tacit in nature.

This paper is made up from 6 chapters. In the 1st section, we mentioned object of the paper, purpose and the background. In the 2 nd section, we will perform an analysis of previous researches of AC with a focus on small and medium-sized manufacturing companies in Japan. In the 3rd section, we will explain a framework of this research. In the 4th section, we will do the evaluation and analysis of two AC case studies by a patent map which is the framework of this research. One is Ball Semiconductor case that does not result in commercialization of a maskless lithography system, the other is PMT case that is successful for the commercialization. In the 5th section, we will explain the implications of in when you aim at innovation which has a core of external technology, and conclusion in the 6th section.

\section{THE PREVIOUS WORK ABOUT ABSORPTIVE CAPACITY}

For $A C$, since there are many good quality review papers, I describe some of the previous research about the importance of $A C$ which pay attention to small and medium-sized manufacturing companies in Japan.

According to the analysis of a company survey, in terms of manufacturing industry, Kodama (2010) states that the development of product type SMEs which has design and sales competences of in-house manufactured goods that has much research and development result such as patent application or new product development and they has power of utilizing industryuniversity cooperation, cooperation with a big firm, and cooperation with SMEs in research and development result. The ability to effectively apply external technology or scientific knowledge 
with other universities and other firms is called AC, and just by having the ability for external knowledge and technology to be utilizable, it is supposed that they can have needs which tackle industry-university cooperation and cooperation between companies positively. Motohashi (2005) also supposes that existence of AC which performs additional technical development which takes in fundamental knowledge of a university in the innovation process in the company for a success of industry-university cooperation that is important in the R\&D-oriented company. Hosoya (2013) examined a survey of manufacturing niche top companies in Japan and found a background that they have been maintained to a high level of AC compared to other SMEs through continuation of business and product development.

As a way to enhance AC, since the research and development that was assisted publicly produce a learning effect, the company will enhance the ability to obtain the latest scientific and technical knowledge (Okada and Kushi, 2004).

In industry-university cooperation from above previous researches, we have found importance of $A C$ and the enhancing method of $A C$ in the organization. The preceding studies conceptually show importance of the high $A C$ for the result of cooperative project such as industry-university collaboration. This has inhibited to be analyzed based on a framework for the AC to SMEs poor management resources to increase the success probability of industryuniversity collaboration. In this paper, we aim at the proposal of a tool for solving this problem. The following section develops our AC evaluation framework.

\section{ABSORPTIVE CAPACITY EVALUATION FRAMEWORK}

Many of the previous researches have set up R\&D expenditure as a proxy variables of $A C$. However, with most of SMEs, the acquisition of $A C$ is performed implicitly to large extent (Vinding, 2006), in addition, R\&D activity is performed in private in many cases (Muscio, 2007). As well as Muscio(2007), we focus on human resources, that is, we evaluate the AC based on the experience level of the members of the organization more directly. Although the AC of organization is not a simple sum of the organization member's AC (Cohen and Levinthal ,1990), considering that the organizational learning be realized only through personal learning (Nicolini and Menzar, 2007), besides, the knowledge in SMEs strongly depend on the person characteristics, we think that there is a certain reasonableness in this approach.

Decision-making on the introduction of external knowledge (technology) is performed subject to a fixed knowledge set unit at the time. In other words, it is natural to think that the product concept is formulated at least when considering the introduction or business utilizing any such knowledge. And by thinking in combination technical resource of its company with technology under consideration, it will be judged whether innovation which utilized external knowledge is realizable. Therefore, in order to construct metrics of AC, a higher level abstract concept, as a measure "readiness,' we should associate with the specific product concept.

Although it is assumed that it is fully recognized in the company about inside information, and it can be accessed in the previous work of AC (Tu et al., 2006), since the maldistribution of knowledge has taken place as mentioned above in SMEs, there is also a case which is hard to be referred to as that the internal knowledge base is recognized enough by the decision maker. Therefore, subjective judgment of the president who is a decision maker in many cases has always 
conceived a possibility of becoming a thing based on recognition of an inaccurate knowledge base. In addition, if the judgment is not based on a reasonable understanding of the knowledge base of its company, it leads to challenge which does not suit the company's capacity, and the risk of jeopardizing the survival of the company is higher. Thus, assuming a company's knowledge base, by the setting of the benchmark to judge whether the introduction of a concerned knowledge set is permissible, we think that we can promote outside-in open innovation of SMEs by supporting the decision making to introduce appropriate outside knowledge into SMEs.

All the industrial products consist of several different engineering components. For example, the main technical component of a liquid crystal panel is constituted by the combination of many technical components like method of display mode (For example, multi domain vertical alignment (MVA)), active element (for example, thin film transistor (TFT)), light volume controller (for example, polarizing plates) and display driver (for example, traffic light controller). In this paper, we propose a method that evaluate AC by decomposing a concerned knowledge set into technical components. By decomposing in this way, even SMEs without the knowledge base for evaluating knowledge systematically, they can consider their AC for external knowledge set under consideration and will be able to evaluate more objectively. However, decomposition into the technical components of the product is difficult if not experts in the product and service. So, this paper shows how to use a citation and quotation patent map by Patent Gazette, as a method of decomposing unfamiliar knowledge set into the technical components mechanically. Many previous researches have described the necessity for the patent map in patent analysis. For example, it is said that in order to remind the intellectual property strategy of their company by recognizing a technical trend as a fingerprint like an illustration, and what is called a technical fingerprint, and grasping it from patent information (Kiriyama, 2009). However, this paper is the first trial to utilize a patent map as an evaluation tool of $A C$, as far as we know.

First, as a method of decomposing into the technical components mechanically for a licensing-in patent, Patent Gazette which patent examiners quoted in the examination process "for patent assessment", are considered to be the technical components which constitutes the patent. Then, as a way to mechanically identify main technical components from the quote Patent Gazette of those (technical components), a technological contents of the Patent Gazette are cited with a large number of other Patent Gazette that is considered to be important components of the technical license. Therefore, it is possible to find out main technical components for the technical license mechanically by performing the sorting in descending order of number of citations for the quote Patent Gazette (technical components).

Next, we evaluate by "Excellent> Good> Average> Bad" for own AC of main technical components which were extracted. The basic idea is in the definition of Cohen and Levinthal (1990) about each technical component, it means that $A C$ belonging to an individual about concerned technical elements is enhanced whenever we go a step, such as "recognize", "assimilate" and "apply".

In addition, it is where there is debate about whether more than the number of citations is made into as the main technical components, but we should assess all reviews if the number of the quoted Patent Gazette (technical components) is less than 10. However, since those technical components will become in redundant if it becomes 100 or more, about the top $10 \%$ is considered to be appropriate. 
Although the previous research has presented many factors as variables for the each construct as "recognize", "assimilate", and "apply" (Jean-Pierre Noblet et al., 2011), when we consider the effectiveness as a decision-making tool in SME, the simplicity of measurement is also important. Based on the above, each evaluation criteria is defined as follows.

- IfSMEs have a track record of product development using the technical component, it should be evaluated as "Excellent".

- If SMEs have the special engineer who takes charge of the technical component, or made a related research paper and related patent application, it should be evaluated as "Good".

- If SMEs have engineers to understand the technical component, it should be evaluated as "Average".

- If SMEs do not have the technical component engineer, it should be evaluated as "Bad".

Finally, as an evaluation index, each evaluation of "Excellent", "Good", "Average" or "Bad" will be given points of 100, 75, 50 and 25 respectively, then obtain an average score. In case of the higher score, it is estimated that AC of its company to perform the licensing-in patent is higher, and it is expected that the industrialization achievement probability of commercialization will be higher. Thus, it is possible to quantitatively evaluate the AC of its company using the patent map for quotation \& citation by the Patent Gazette in this way. The AC evaluation procedure for each technical component with the mentioned above patent map is shown in Table 1.

Table 1 Absorptive Capacity evaluation procedure

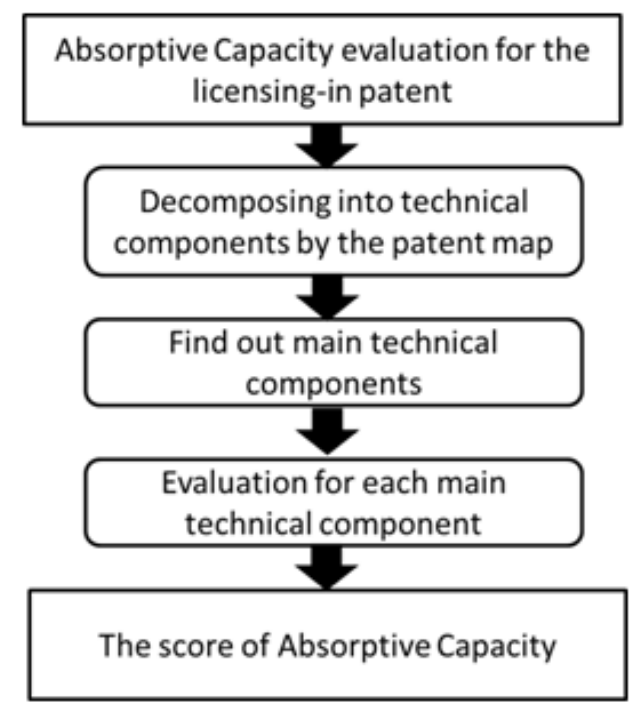

First of all, without ability to search of the promising external technology, we cannot perform innovation that utilized external technology. Although the framework of this paper is utilizable also for evaluation of $A C$ in the viewpoint of recognition of a certain external knowledge group, the evaluation of cognitive power to each external technical set requires a multi-faceted discussion, such as a method for a knowledge group definition. In this paper, since we have focused on AC as a measure of readiness for specific external knowledge set, we would like to suppose to hand over the evaluation framework of recognition relates to "recognize" to another paper, and we will define the external knowledge set under consideration in this paper, we will show how to apply the framework in the next section. 


\section{CASE: MASK-LESS DRAWING EQUIPMENT}

The use of the AC evaluation method described above is illustrated by comparing the two companies for which it was going to realize the same product innovation by taking in external technology from the same university researcher by practical use of industry-university cooperation.

Mr. Ishikawa who was former vice president of Texas Instruments, Inc. (TI) founded the Ball Semiconductor, Inc. (Ballsemi) at Allen in Texas, U.S., in October, 1996, with \$US 52 million of capitals, 50 employees, and develop and manufacture spherical semiconductor sensors, medical device and photovoltaic device.

PMT Corporation (PMT) is the SMEs in Japan, \$US 500,000 of capitals, and about 100 employees, it starts from a trading company, and becomes a research-and-development type company through outside-in open innovation by industry-university cooperation, and major business is the manufacture of a semiconductor manufacturing related equipment, ultraprecision axis control related equipment etc..

\section{Case Outline}

It is necessary for various process technologies in semiconductor manufacturing, the semiconductor technology has been making drastic progress by lithography process to do microfabrication in particular. The exposure equipment transfers an integrated circuit on a wafer in the lithographic process. The exposure equipment performs the transfer in reduction projection onto a wafer by using a mask drawing a circuit pattern obtained by dividing each layer integrated circuits. The number of masks required for one kind of semiconductor manufacture needs 20 to 40, and mask cost for a submicron or a nanometer patterning semiconductor is about \$US 10 million to \$US 100 million, even at the micron level, the mask cost is some \$US 10,000 . Furthermore, it is needed long lead-time that is 1 to 3 months. High running costs are required to maintain the mask in this way.

In order to solve this issue, CEO/CTO Ishikawa of Ballsemi had an idea that used TI DMD (Digital Micro- mirror Device) chip instead of the mask, because he had a profound knowledge of DMD when he was vice president of TI. He conducted industry-university cooperation with a professor of Tohoku University to develop a mask-less drawing technology with DMD, and he performed the patent application with Tohoku University. This does not need a mask and transfers a circuit pattern to a DMD chip and draws a circuit pattern on a wafer with a reduction projection lens by scanning $X-Y$ stage in sync with the transfer speed (Figure 1). However, this equipment development did not lead to up to commercialization.

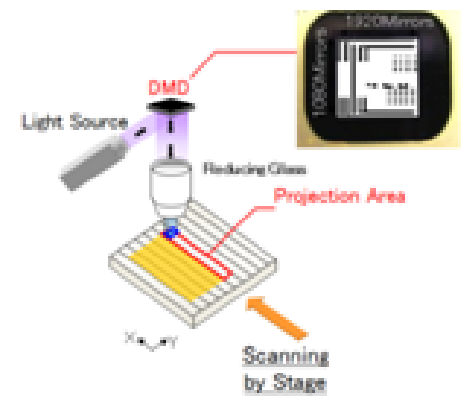

Figure 1 The mask-less drawing technology with DMD 
Figure 1 The mask-less drawing technology with DMD

On the other hand, PMT has acquired an ultra-precise XYZ stage control technology with a resolution of the nano-level performance through the "development of micro-nano fabrication system" by METI (Ministry of Economy, Trade and Industry) regional consortium. In addition, a researcher who has a PhD in this field was also joined.

The professor had continued research and development even after the end of joint research with Ballsemi, and PMT has been able to achieve commercialization of the mask-less drawing equipment with Tohoku University by taking advantage of public subsidy (supporting industry program) that is "Research and development of pattern generation technology and positioning accuracy of the microfabrication device ". In this way, originally Ballsemi started innovation of the mask-less drawing equipment with a researcher of Tohoku University and let PMT succeed it in licensing-in later and has achieved commercialization. The interest of this paper is to explain why Ballsemi was forced to abandon the commercialization of joint research results, while PMT was able to come out. We will analyze and evaluate the difference in AC of both companies with the citation and quotation patent map by Patent Gazette which is the framework of this report.

Figure 2 is a quote-cited relationship diagram of Japanese Patent Laid-Open No. 20051567788 of mask-less lithography system that Tohoku University has been filed. Table 2 shows the number of quotes-citations in Japanese Patent Laid-Open No. 2005-1567788. As can be seen on the patent map, the patent examiner referred to 246 Patent Gazette in order to determine "refusal" or "patent assessment". As for that we can say, the mask-less drawing equipment is constituted by very much inventive technical components. In other words, it is expected that the commercialization is very difficult if AC of these technical components is lacking.

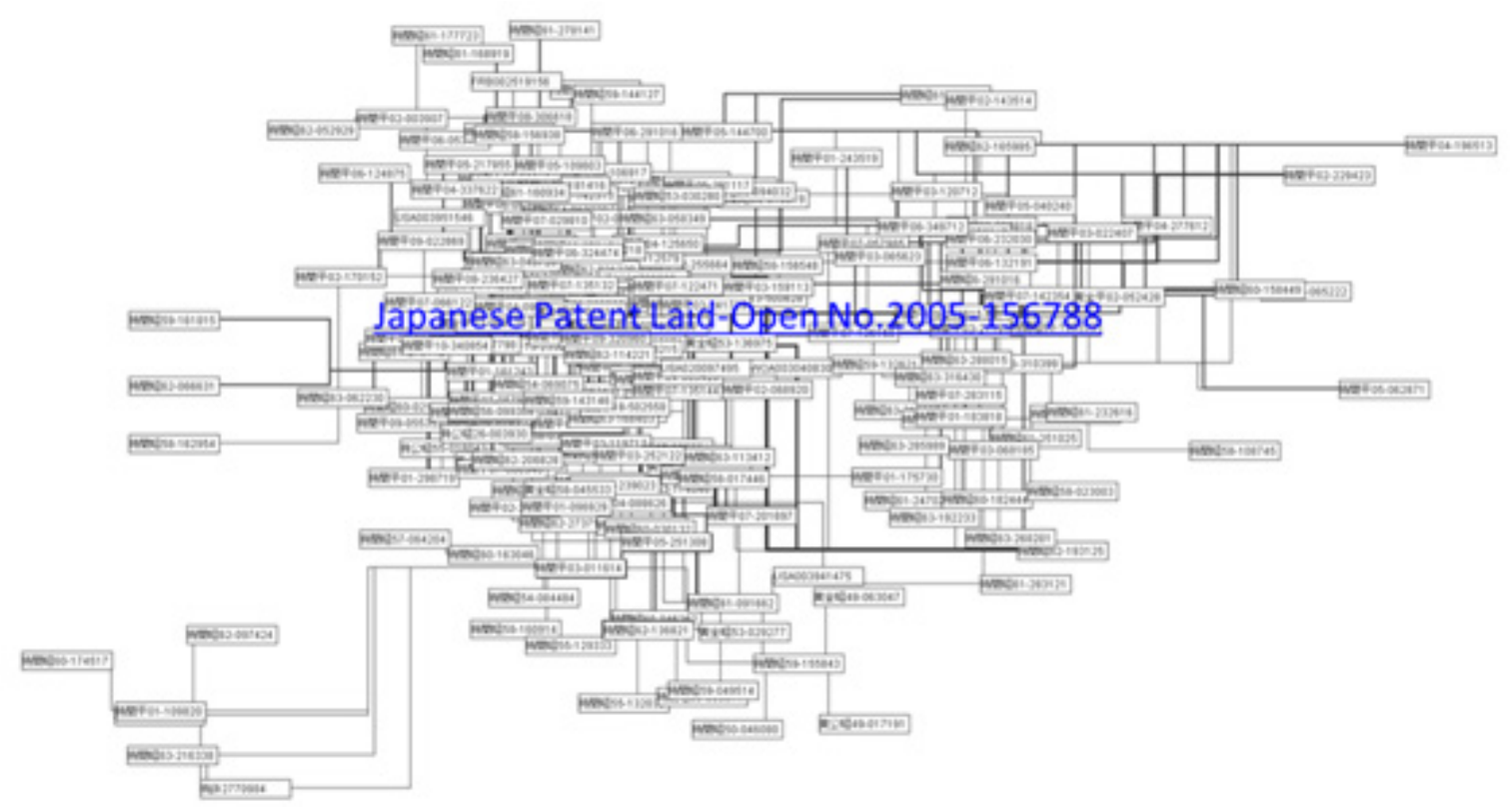

Figure 2 The quote-cited relationship diagram of Japanese Patent Laid-Open No. 2005-1567788 Data source: YUPASS (Yamaguchi University Patent Search System) 
Table 2 The quotation \& citation patent map for Japanese Patent Laid-Open No. 2005-1567788

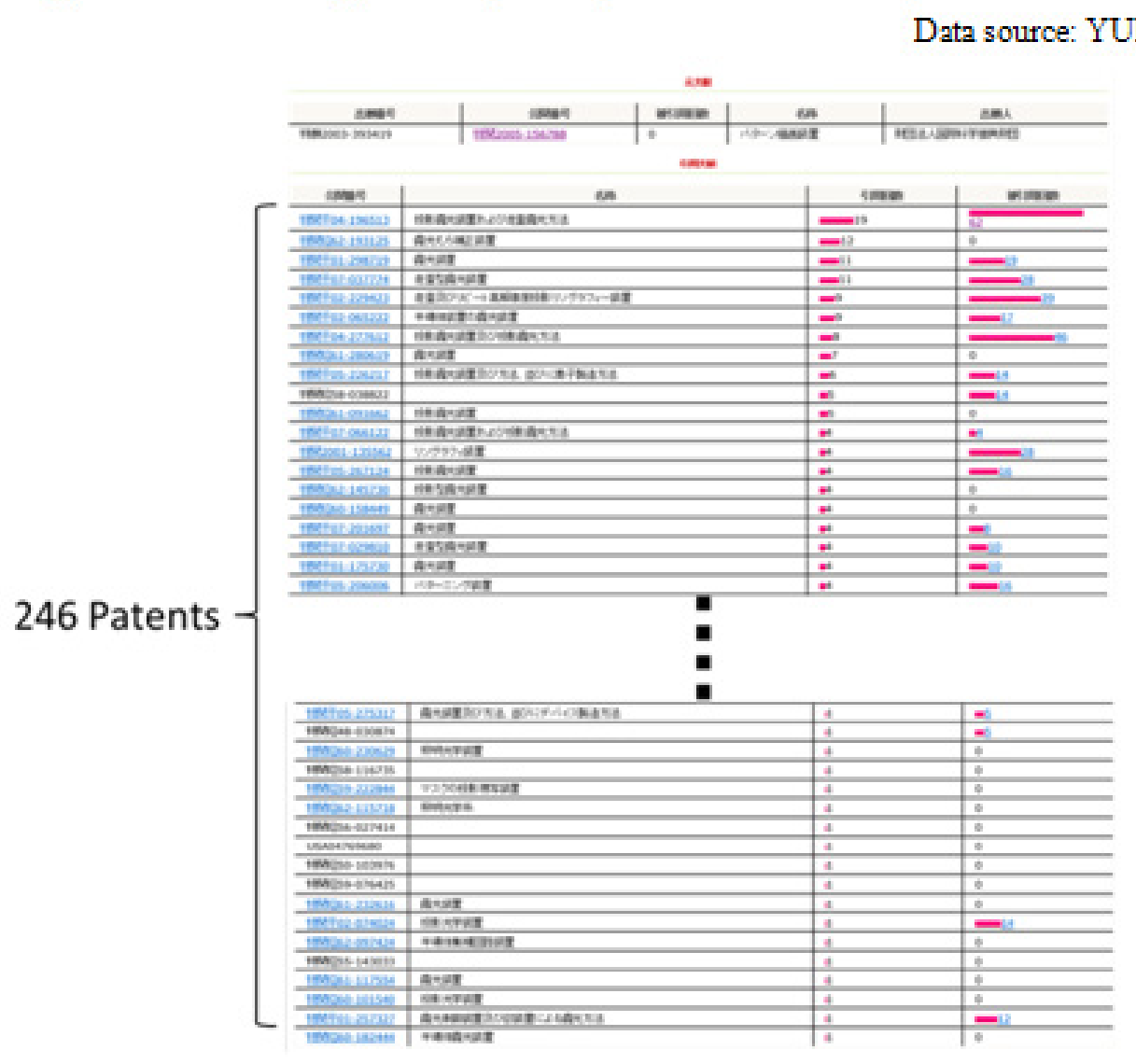

\section{Implementation of the AC evaluation framework}

Since Patent Gazette with much number of citations is generally a fundamental patent for the invention, we suppose that the mask-less lithography system is possible to utilize technologies described in these publications. The roughly top $10 \%$ that is referenced more than 15 times are supposed having important technical components that are indispensable for the mask-less drawing equipment, and as shown in Table 3, we suppose technical component of the mask-less drawing equipment by analogy from the technical component of each Patent Gazette. For each technical component, based on the interview with Ballsemi and PMT, we evaluate as "Excellent>Good>Average>Bad" with the criteria mentioned above. Finally, for each and adding points $100,75,50,25$, to obtain an average score. As a result, the metrics of the AC for Bollsemi is 46.9 points, while PMT is 73.4 points was found.

PMT has achieved the commercialization of the mask-less drawing equipment due to highly AC of each technical component, on the other hand, since Ballsemi was insufficient for $A C$ of the ultra-precision moving control technology for the stage which synchronized with the pattern data transfer speed which is the most important technical component of the mask-less drawing equipment, we suppose that it did not technically lead to the product development. In addition, Ballsemi licensed out the technology to a company in Japan, which successfully commercialized. 
Table 3 The AC rating for each technical component of the mask-less lithography system

\begin{tabular}{|c|c|c|c|c|c|c|c|c|c|c|c|}
\hline \multirow{3}{*}{$\begin{array}{c}\text { Ma } \\
1\end{array}$} & \multirow{3}{*}{ 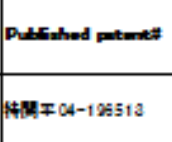 } & \multirow{3}{*}{ 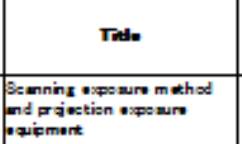 } & \multirow{3}{*}{ Aprome } & \multirow{3}{*}{\begin{tabular}{r|} 
of \\
onotention \\
12
\end{tabular}} & \multirow{3}{*}{ 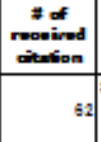 } & \multirow{3}{*}{ 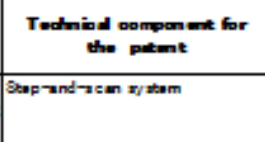 } & \multirow{3}{*}{ 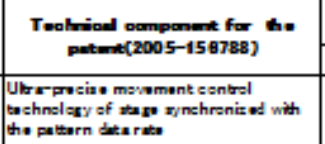 } & \multicolumn{4}{|c|}{ Absoption Cypaty } \\
\hline & & & & & & & & \multicolumn{2}{|c|}{ BAlnmini } & \multicolumn{2}{|c|}{ PMT } \\
\hline & & & & & & & & Ead & 25 & Eved dent & 100 \\
\hline 2 & 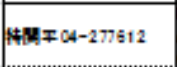 & 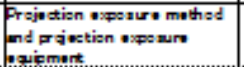 & Mksen & 8) & 43 & 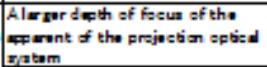 & 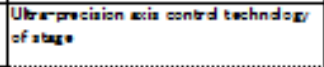 & Ead & 25 & Eved dent & 100 \\
\hline$a$ & $1402+02-2220423$ & 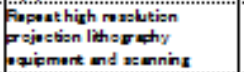 & Kantilel by & 2] & $a a^{8}$ & Ptap-and-aten x/tam & 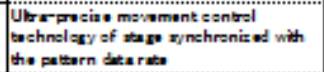 & Ead & 25 & Evedlone & 100 \\
\hline 4 & $1404+07-037774$ & Bemning ovpo wre equipment & Comnan & 11 & $28 \mid$ & 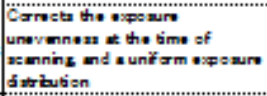 & Enfecto Momer Mue & Aererase & 50 & Goed & 75 \\
\hline 5 & \$ $142001-135952$ & Pthesrathic equiment & Hiksehi & 4 & 28 & Mentione eqpesure methed & DMO emeral todinalar & Good & 75 & Good & 75 \\
\hline 6 & 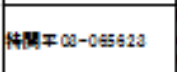 & 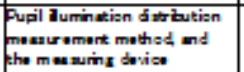 & Hìnati & 1 & $25 \mathrm{~m}_{\mathrm{m}}^{\mathrm{m}}$ & 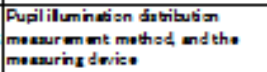 & Ë̈estion Mamer Mas & Gaod & 75 & Good & 75 \\
\hline 7 & $1942705-251308$ & $\begin{array}{l}\text { Method for fobricatha s } \\
\text { semico nduetor device }\end{array}$ & Mksn & a) & $24=$ & $\begin{array}{l}\text { Hidh-porf ammense ightins aptied } \\
\text { xyatam }\end{array}$ & 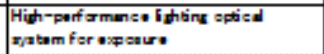 & Ead & 25 & Bad & 25 \\
\hline$a$ & $1404=06-310320$ & 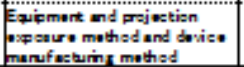 & Hesen & 2 & 20 & Eipo wure eserectian methed & 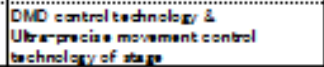 & Aererase & 30 & Good & 75 \\
\hline 2 & 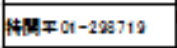 & Eqpaure deviou & 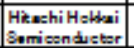 & 11 & 102 & Autemetisn of lishting equatoment & $\begin{array}{l}\text { Lutsmatad a mara bisn off ectia Mira } \\
\text { Mes }\end{array}$ & Eererass & 30 & Goed & 75 \\
\hline 10 & 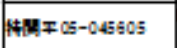 & Fryteing fó:d devise. & Pentax & 2 & 120 & 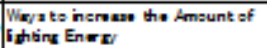 & 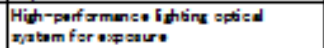 & Ead & 25 & Bed & 25 \\
\hline 11 & $1+42 \mp 07-057986$ & Fonse methed and & Mksn & 2 & is & 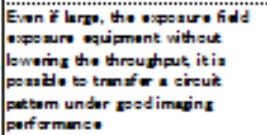 & 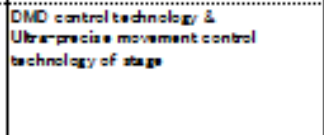 & Aererase & 30 & Good & 75 \\
\hline 12 & $1+1242000-053442$ & 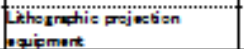 & LSLM & a) & 17 & Ürifom inte diatribution & 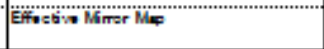 & Aererases & 50 & Goed & 75 \\
\hline 13 & $1402702-065222$ & 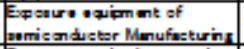 & Yumasza NEC & 2 & $17]^{2}$ & 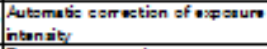 & 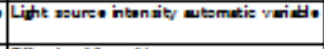 & Good & 75 & Good & 75 \\
\hline 14 & $1404=06-232030$ & 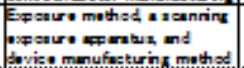 & Niken & 2 & 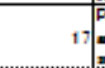 & 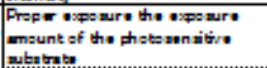 & 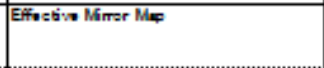 & ererases & 50 & Good & 75 \\
\hline 15 & $15124+05-206006$ & Fattarrins equicmert & $\begin{array}{l}\text { Taxas } \\
\text { Instrumente }\end{array}$ & 4 & 16 & Drwing dovice of sterand- & HA & HA. & HA & HA. & nA. \\
\hline 16 & $1+42705-267124$ & Frajectisn wopeaure & Touliba & 4. & 16 & impowem me of the fitar dofining & 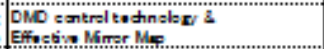 & erenase & 50 & Goed & 75 \\
\hline 17 & 1\$4 $706-324474$ & $\begin{array}{l}\text { Fastama akend } \\
\text { Expauro methed }\end{array}$ & Niken & 2 & $=$ & 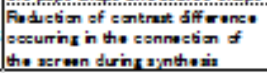 & 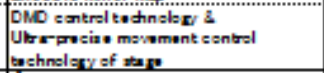 & feverase & 50 & Good & 75 \\
\hline & & & & & & & Ave Polnt & & पर्ब & & 73.4 \\
\hline & & & & & & & $\begin{array}{l}\text { Bed: } 25, \text { Avaras: } 50 \\
\text { Cosd: } 75 \text {,Eved ant: } 100 \text { paint }\end{array}$ & & & & \\
\hline
\end{tabular}

\section{IMPLICATIONS}

When aiming at an innovation which used external technology as the core, with AC of its company by evaluating on the quotation \& citation patent map which is a framework of this research, it is possible to build an associate base of the management judgment such as licensingout, licensing-in, outsourcing-in, partnership networking, etc..

Even if we carry out technical search and learning of the collaborative investigation and then created some kind of knowledge, the subsequent activity is not connected directly to the conclusion whether you should carry out in-house. When AC is insufficient like Ballsemi, a management judgment which carries out licensing-out to the other company can be attractive. Ballsemi acquired the loyalty by it, and the company which did licensing-in has succeeded in commercializing.

On the other hand, you can perform a management judgment to enforce licensing-in when you have enough AC to its commercialization as PMT. In addition, if there is a technical component which is insufficient of $A C$, you should not give up R\&D or commercialization, you should consider a management judgment which complement the lack of AC with open innovations, such as outsourcing-in and partnership networking, can also be made. Since PMT did not have an optical technology, the development of the technology was assigned to the 
external optical special marker. However, since an integration, such as adjustment, was required in order to incorporate an optical system in the equipment, currently PMT has employed optic engineers in the company.

\section{CONCLUSION}

When the product development type SMEs, where R \& D resource is scarce, performs a licensing-in of a seed which a university or a research institution have, and commercialize with a unfamiliar technical component by industry-university cooperation, AC which assimilates and takes the technical component into its company is required. As a method to evaluate this, we extracted major technical component by the quotation \& citation patent map by Patent Gazette and showed the method to evaluate the company's AC for it through the case study of the maskless drawing equipment of Ballsemi and PMT.

If the evaluation score is low, the commercialization can be made by performing the licensing-out to other companies as example of Ballsemi. In addition, when an evaluation score of a technical component is low, neither development nor commercialization can be given up but a management judgment compensated with the lacking technology by licensing-in etc. can also be made.

However, when making the decision to embrace external knowledge, it does not necessarily mean that we should only take an external knowledge group that scores high. A high score suggests the height of the technical vicinity of external knowledge to company knowledge base, and suggests the height of the feasibility of commercialization. On the other hand, the decision to accept only high score promotes conservative posture against external knowledge as it implies to accept what is familiar and is at risk of inhibiting learning about external new knowledge. Therefore, in order to aim at the expansion of AC (Zahra and George, 2002) as a dynamic capability that affect the sustainability of the company's competitive advantage, you need to have a variety to the score of the external knowledge acquisition project which exceeds the lower threshold of AC designated in the company. A strategic judgment is necessary whether we should try it, even if AC score is low if it is risky yet promising project.

Finally we should show the limit of the approach developed in this report. Needless to say, this paper is exploratory research, and must be validated by the application to many cases. Be premised on it, when the seeds which a university or a public research organization create have limited path dependency from existing technical component, since there are few numbers of Patent Gazette which a patent examiner quotes, it is difficult to extract main technical components through the quotation \& citation patent map. In addition, the evaluation method of this report is not applicable when techonologycal componets of the seeds are unobservable. In addition, even if we have the given conditions which is the validity of attention to individual, we must consider the need to consider the influence on AC such as individual learning ability, motivation, and personality. We would like to make these points in a future discussion. 


\section{REFERENCES}

1. Henry Chesbrough, OPEN INOVATION The New Imperative For Creating and Profiting From Technology, HARVARD BUSINESS SCHOOL PRESS, Boston, 2003.

2. Kazuyuki Motohashi, Yohji Ueda, and Motoharu Mieno, The new trend on the open innovation of Japanese companies: Results and Discussion of the interview survey of leading manufacturer, RIETI Policy Discussion Paper Series, 2012, 12-P-015 (In Japanese).

3. Veugelors, R., and Cassiman, B., Make or Buy in innovation strategies: evidence from Belgium manufacturing firms, Research Policy, 1999, 28 (1), 63-80.

4. Wesley M. Cohen and Daniel A. Levinthal, Innovation and Learning: the Two Faces of R\&D. The Economic Journal, 1989, Vol.99, pp. 569-596.

5. Wesley M. Cohen and Daniel A. Levinthal. Absorptive Capacity: A New Perspective on Learning and Innovation. Administrative Science Quarterly, Vol. 35, No. 1, Special Issue: Technology, Organizations, and Innovation, 1990, pp. 128-152.

6. Lane, P. J., Koka,B.R, and Pathak. S., The reification of absorptive capacity: A critical review and rejuvenation of the construct. Academy of Management Review, [2006, 31 (4) 833863.

7. Volberda, H.W, Nicolai J. Foss, N.J. and Lyles, M.A, Absorbing the Concept of Absorptive Capacity: How to Realize Its Potential in the Organization Field, Organization Science, 2010, 21 (4), 931-951.

8. Shaker A.Zahra and Gerard George, Absorptive Capacity: A Review, Reconceptualization, and Extension. Academy of Management Review, 2002, Volume 27, Issue 2, pp. 185-203.

9. Todorova, G., and Durisin. B., Absorptive capacity: Valuing a reconceptualization. Academy of Management Review, 2007, 32 (3), 774-786.

10. Kira R. Fabrizio, Absorptive capacity and the search for innovation, Research Policy, 2009, 38, 255-267.

11. Nika Murovec and Igor Prodan, Absorptive capacity, its determinants, and influence on innovation output: Cross-cultural validation of the structural model Technovation, 2009, 29, 859-872.

12. Vinding, A.L., Absorptive capacity and innovative performance: a human capital approach. Economics of Innovation and New Technology, 2006, 15 (4/5), 507-517. 
13. Becker, W., Dietz, J., R\&D cooperation and innovation activities of firms-evidence for the German manufacturing industry. Research Policy, 2004, 33 (2), 209-223.

14. Van Den Bosch, F.A.J., Volberda, H.W., De Boer, M., Coevolution of firm absorptive capacity and knowledge environment: organizational forms and combinative capabilities. Organization Science 10(5), 1999,551-568.

15. Mark Dodgson and Roy Rothwell, The Handbook of Industrial Innovation, Aldershot Hants: Edward Elgar, 1994.

16. Noteboom, B., Innovation and Inter-firm Linkages: New implications for policy, Research Policy, 1998, 28 (4), 793-805"

17. Maskell, P., and Malmberg, A., Localised Learning and Industrial Competitiveness. Cambridge Journal of Economics, 1999, 23 (4), 167-185.

18. Madrid-Guijarro, A., Garcia, D., and Van Auken, H. 2009, Barriers to Innovation among Spanish Manufacturing SMEs. Journal of Small Business Management, 47 (4), 465-488.

19. Toshihiro Kodama, Empirical studies indicating the possibility of formation of industrial clusters centered on product development SMEs, RIETI Policy Discussion Paper Series, 2010, 10P-030 (In Japanese).

20. Kazuyuki Motohashi, R \& D network and industry-university cooperation of SME: The positioning of innovation system in Japan in the period of change, RIETI Policy Discussion Paper Series, 2005, 05-J-002 (In Japanese).

21. Yuji Hosoya, Study of manufacturing small and medium-sized companies that have excellent represented by global niche top: Mainly a questionnaire survey of manufacturing niche top companies in Japan, RIETI Discussion Paper Series, 2013, 13-J-007 (In Japanese).

22. Yosuke Okada and Takahito Kusi, Joint research and development and government: Funded business Case study of drug mechanism ventures, Office of Pharmaceutical Industry Research, 2004, No.16 (In Japanese).

24. Muscio, A., The impact of absorptive capacity on SMEs' collaboration, Economics of Innovation and New Technology, 2007, 16 (8), 653-668.

25. Nicolini, D., and Meznar, M.B., The social construction of organizational learning: conceptual and practical issues in the field. Human Relations, 1995, 48 (7), 727-746. 
26. Qiang Tu, Mark A. Vonderembse, T.S. Ragu-Nathanb, and Thomas W. Sharkey Absorptive capacity: Enhancing the assimilation of time-based manufacturing practices, Journal of Operations Management, 2006, 24, 692-710.

27. Patent application technology trends survey report: Image quality improvement technology of liquid crystal display device, Patent office, 2005 (In Japanese).

28. Tsutomu Kiriyama, Philosophy path of patent analysis and analysis, Information management, 2009, Vol.52 no. 5, pp. 286-299 (In Japanese).

29. Jean-Pierre Noblet, Eric Simon and Robert Parent, Absorptive capacity: a proposed operationalization, Knowledge Management Research \& Practice (2011) 9, 367-377. 